\title{
Green synthesis of silver chloride nanoparticles using Rhodotorula Mucilaginosa
}

\author{
Ioana GHIUȚA ${ }^{1, a^{*}}$, Daniel CRISTEA ${ }^{1, \mathrm{~b}}$, Rodica WENKERT ${ }^{2, \mathrm{c}}$, \\ Daniel MUNTEANU ${ }^{1, d}$ \\ ${ }^{1}$ Transilvania University, Eroilor 29, Brasov, Romania \\ ${ }^{2}$ Yitzhack I. Rager Blvd 151, Beersheba, Israel \\ a*ioana.ghiuta@unitbv.ro, bdaniel.cristea@unitbv.ro ${ }^{\mathrm{C}}$ RodicaWe@clalit.org.il \\ ddanielmunteanu@unitbv.ro
}

Keywords: Silver chloride nanoparticles, Rhodotorula Mucilaginosa, Green synthesis.

\begin{abstract}
The biosynthesis of silver chloride nanoparticles (AgCl NPs) is presented in this paper. Silver chloride nanoparticles were synthesized using fungi culture from Rhodotorula Mucilaginosa and aqueous $\mathrm{AgNO}_{3}$ solution, as precursor. The plasmon resonance of the nanoparticles containing solution has shown through UV-visible spectrophotometry an absorbance peak at about $437 \mathrm{~nm}$. Scanning Electron Microscopy, Energy Dispersive Spectroscopy, and X-ray Diffraction analyses confirmed the presence of spherical silver chloride nanoparticles with a face centered cubic crystal structure and an average particle size of $25 \mathrm{~nm}$. Silver chloride nanoparticles have been shown to be able to inhibit the growth of different microorganisms, including bacteria and fungi, which would make them suitable for antimicrobial applications.
\end{abstract}

\section{Introduction}

The development of materials and structures at nanoscale dimensions has gained a huge interest in the nanomaterials and nano-technology research fields. One of the most important properties of metallic nanoparticles is their antimicrobial activity.

Eco-friendly methods concerning nanomaterials synthesis present a substantial importance for biological applications, mainly due to nontoxic substances and environmentally friendly procedures employed [1].

Green synthesis of silver chloride nanoparticles has been reported to be mediated by different kinds of organisms, from bacteria to plant extracts. Cell-free culture supernatant of Streptomyces strain, Klebsiella planticola, biomass of Bacillus subtilis, leaf extract of Cissus quadrangularis, aqueous extract of Sargassum plagiophyllum, extract from needles of Pinus densiflora, Prunus persica $L$. outer peel extract are just a few examples of organisms able to synthesize AgCl NPs [2]-[8].

Weili Hu et al. have presented the synthesis of silver chloride nanoparticles under ambient conditions in nanoporous bacterial cellulose membranes as nanoreactors. It has been demonstrated that the synthesized silver chloride nanoparticles exhibited high hydrophilic ability and a strong antimicrobial activity against Staphylococcus aureus and Escherichia coli bacteria [9]. The antibacterial effect of biosynthesized AgCl NPs investigated against Escherichia coli was found to be dose-dependent [6]. The biosynthesized silver chloride nanoparticles exhibited besides the antimicrobial activity, cytotoxicity activity against HeLa and SiHa cancer cell lines [2]. 
M. Sophocleous and J. K. Atkinson have described in their review the significant development of $\mathrm{Ag} / \mathrm{AgCl}$ screen printed sensors [10]. Moreover, $\mathrm{Ag} / \mathrm{AgCl}$ NPs are examined for further applications of nanoparticles as a plasmonic photocatalyst [6].

The reports which have shown the importance of the AgCl NPs application, from sensors, catalysts, to antimicrobial activity, have led to the research results presented herein. Even though several methods to obtain nanoparticles are currently developed, the green methods have captured the interest of researchers due to their lack of toxicity. In the present work, the green synthesis of silver chloride nanoparticles is described. This is, to the best of our knowledge, the first report for green synthesis of AgCl NPs mediated by Rhodotorula Mucilaginosa.

\section{Materials and Methods}

\section{Fungi and culture conditions}

The fungi Rhodotorula Mucilaginosa used in this study were provided from Soroka University Medical Center from Beersheva, Israel. The fungi were cultivated in the solid media, Sabouraud agar supplied by Scharlau Chemicals, and incubated at $35^{\circ} \mathrm{C}$ for $48 \mathrm{~h}$.

\section{Biosynthesis of AgCl nanoparticles using Rhodotorula Mucilaginosa}

In order to synthesize the silver chloride nanoparticles, $1 \mu l$ of bacterial strains were freshly inoculated in test tubes containing $15 \mathrm{ml}$ of growth medium, namely Brain Heart Infusion from Sigma Aldrich. The liquid media contained beef heart (infusion from $250 \mathrm{~g}$ ), $5 \mathrm{~g} / \mathrm{L}$; calf brains (infusion from $200 \mathrm{~g}$ ), $12.5 \mathrm{~g} / \mathrm{L}$; disodium hydrogen phosphate, $2.5 \mathrm{~g} / \mathrm{L}$; D(+)-glucose, $2 \mathrm{~g} / \mathrm{L}$; peptone, $10 \mathrm{~g} / \mathrm{L}$; sodium chloride, $5 \mathrm{~g} / \mathrm{L}$. The liquid culture was kept in a thermostat at $35{ }^{\circ} \mathrm{C}$ for $24 \mathrm{~h}$, followed by centrifugation at $4000 \mathrm{rpm}$ for $30 \mathrm{~min}$. The supernatant and biomass were tested in parallel. In the first situation $5 \mathrm{ml}$ of supernatant was used, while for the second one the biomass was kept with the addition of $5 \mathrm{ml}$ of distilled water. The next step was similar by adding

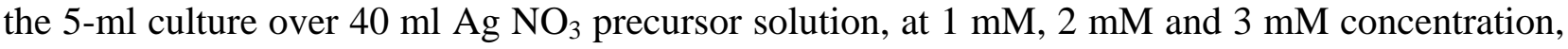
respectively. The culture, supernatant and biomass + distilled water and precursors were kept as control. The samples were kept in a thermostat set at $35^{\circ} \mathrm{C}$, for $48 \mathrm{~h}$.

\section{Characterization of AgNPs}

Ultraviolet-visible spectral analysis was carried-out by using Jasco V-630 spectro-photometer. The UV-visible spectra were measured in the range $200-600 \mathrm{~nm}$ with a wavelength step size of $1.5 \mathrm{~nm}$.

For morphological characteristics and chemical composition, a JSM 7400f scanning electron microscope (SEM) with a platform for Energy Dispersive Spectroscopy (EDS) was used. The silver chloride nanoparticles colloid was dropped on a copper grid and coated with a platinum thin film. After this the samples were mounted on a double sided adhesive carbon-tape. The acceleration voltage was fixed to $10 \mathrm{kV}$.

The crystalline nature of silver chloride nanoparticles was analyzed by XRD using a Philips PW 1050/70 X-ray powder diffractometer with graphite monochromator using $\mathrm{CuK}_{\alpha 1}(\lambda=1,54 \AA)$, at a voltage of $40 \mathrm{kV}$, a current of $28 \mathrm{~mA}$, in the scan range $10 \div 80^{\circ}$, in Bragg-Brentano geometry.

\section{Results and Discussion}

After incubation in the thermostat for $48 \mathrm{~h}$ at $35{ }^{\circ} \mathrm{C}$, the final color of the colloid, containing biomass of Rhodotorula Mucilaginosa, changed from light yellow to light brown. The change in color is an indication for the formation of nanoparticles. In Fig. 1 the obvious difference in color can be observed, depending on the precursor concentration. The samples with supernatant and the control have remained unchanged. Furthermore, considering the optical indication (changing the color), the UV-visible absorption spectra of the colloidal solutions with nanoparticles was measured (shown in Fig. 2). 


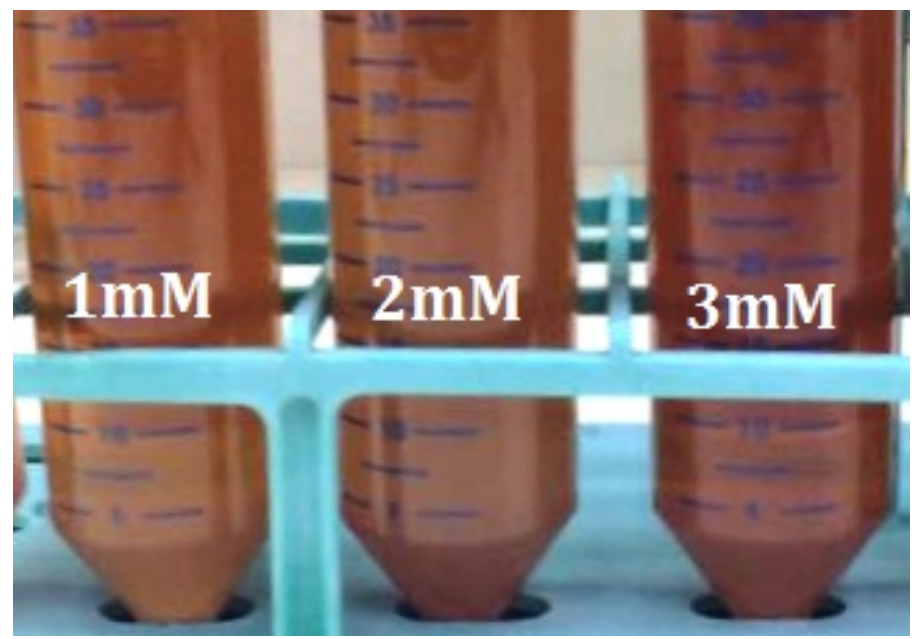

Fig. 1. Image of colloidal nanoparticles synthesized in the presence of Rhodotorula Mucilaginosa.

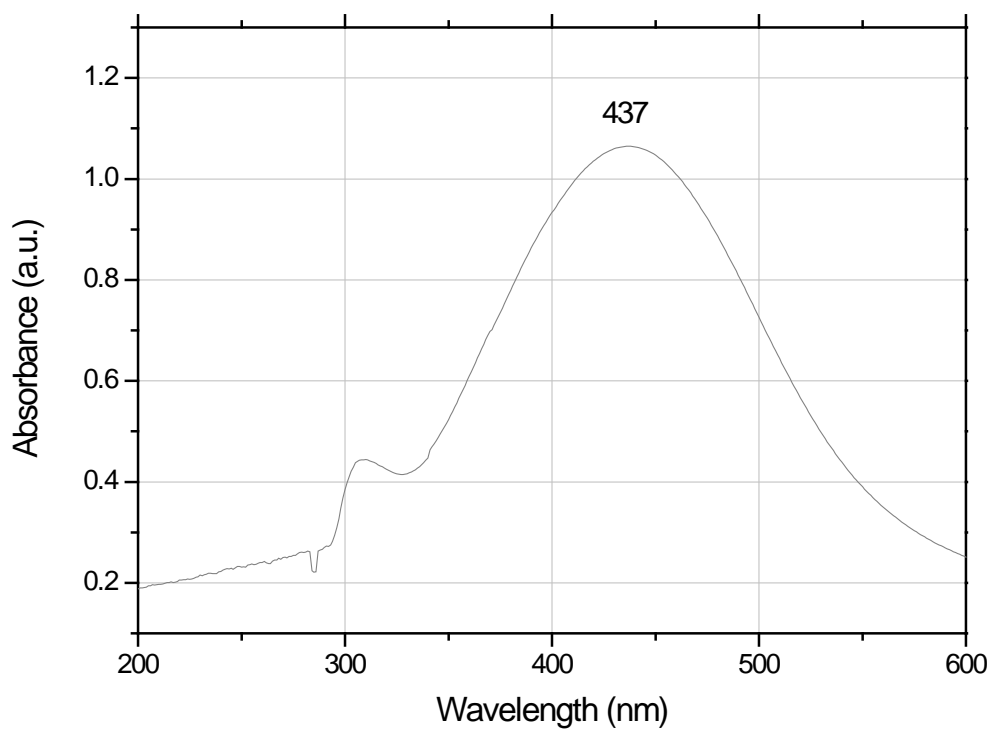

Fig. 2. UV-visible spectrum of the AgCl nanoparticle containing colloids3.

Fig. 2 corresponds to the UV-visible absorption spectrum of the solution containing AgCl NPs synthesized using biomass of Rhodotorula Mucilaginosa fungi. The spectra showed the maximum absorbance at $437 \mathrm{~nm}$ for samples with concentration $3 \mathrm{mM} \mathrm{AgNO}_{3}$. Several reports have provided similar results concerning the absorption spectrum. The reports have confirmed that peaks around $440 \mathrm{~nm}$ coincide to the plasmon resonance of silver chloride nanoparticles [4], [5].

The crystalline nature of synthesized nanoparticles, obtained from X-ray diffraction, is confirmed by the diffraction peaks shown in Fig. 3, which correspond to the (111), (200), and (220) planes of the face centered cubic structure of AgCl crystal (Fig. 3) [11]. 
An explanation for the presence of silver chloride nanoparticles can be based on the interaction between silver nitrate and bacteria, which was previously grown in Brain Heart Infusion Broth media containing sodium chloride.

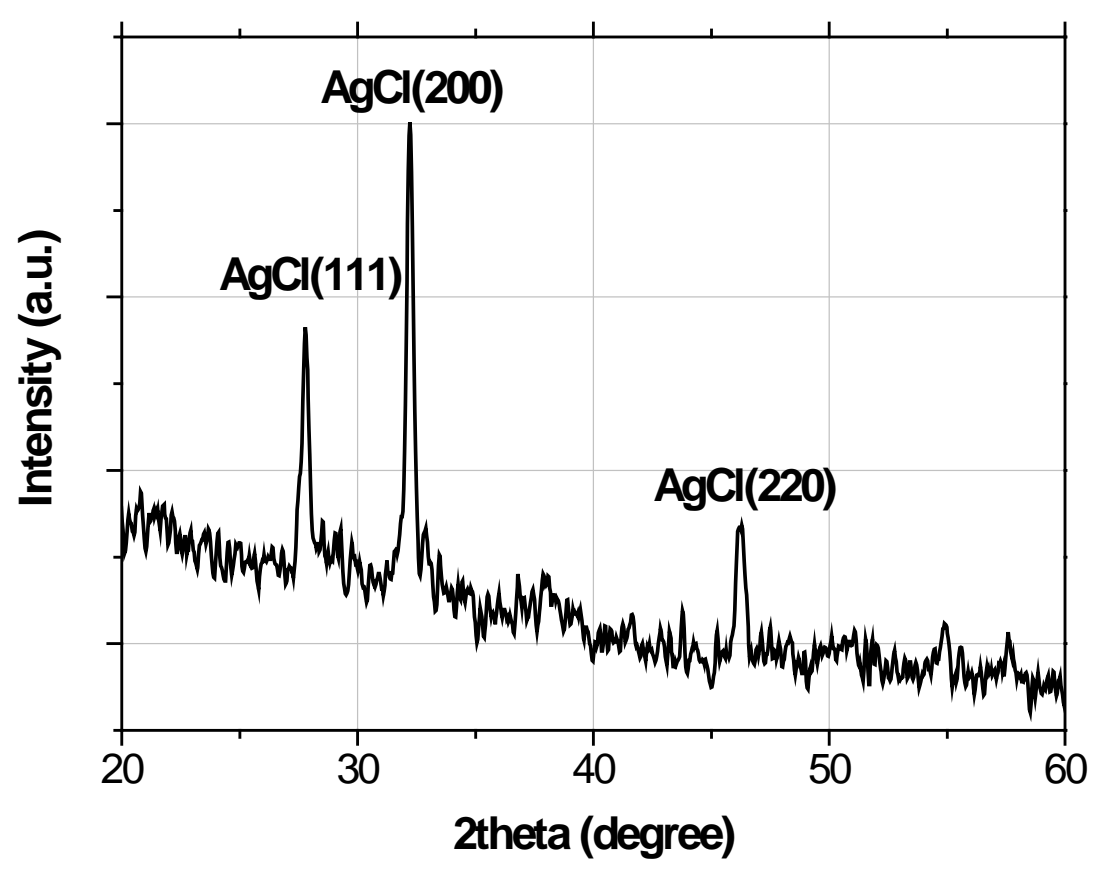

Fig. 3. X-Ray Diffraction pattern for AgCl nanoparticles.

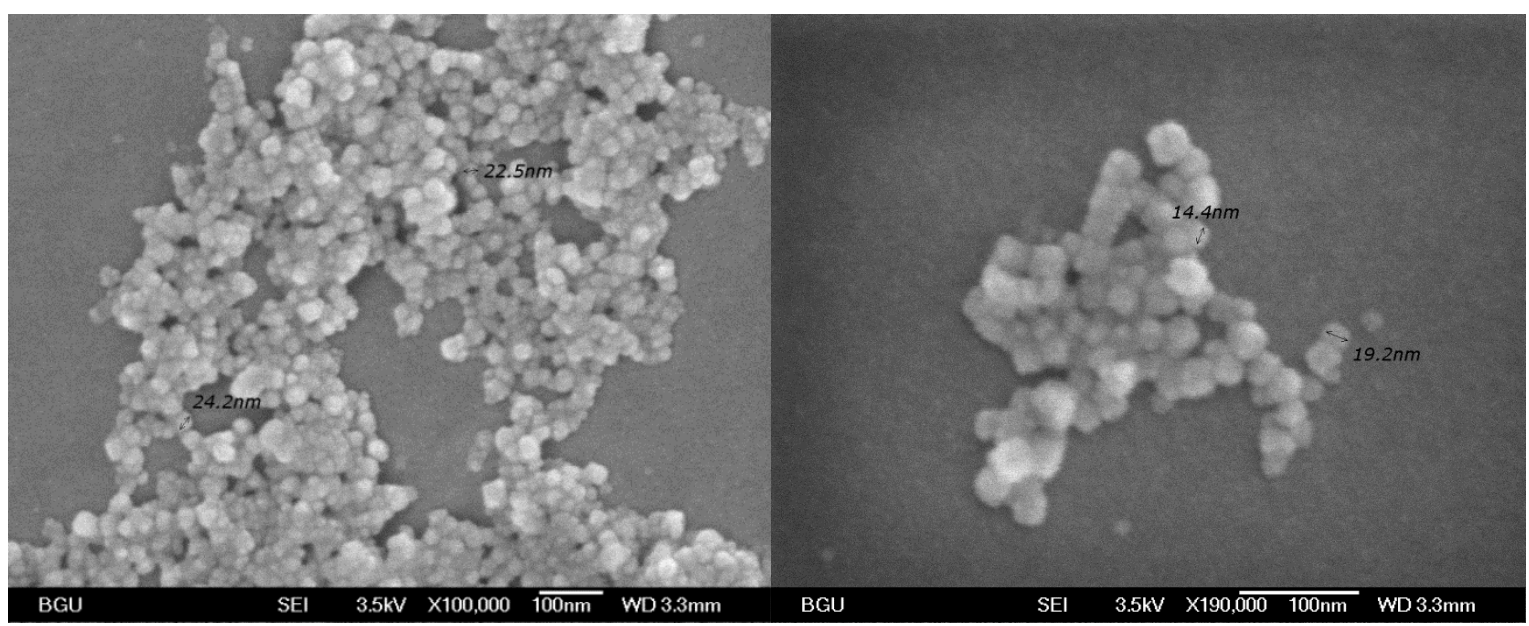

Fig. 4. SEM images of silver chloride nanoparticles at $1 \mathrm{mM}$, and $3 \mathrm{Mm} \mathrm{AgNO}_{3}$, respectively. 
The SEM analysis (Fig. 4) confirmed the presence of nano-scaled particles and also showed the spherical shape of them. As can be seen on the SEM micrographs, the silver chloride nanoparticles have dimensions smaller than $25 \mathrm{~nm}$. The particle size/antimicrobial efficacy relation was reported previously, smaller particles, due to their much larger surface area, are expected to exhibit a much higher antibacterial efficacy.

The EDS spectra presented in Fig. 5 show the presence of the principal elements, namely Ag and $\mathrm{Cl}$. The EDS analysis also revealed others elements which can be found on the samples due to the preparation stages (copper grid, carbon tape, thin film of platinum).
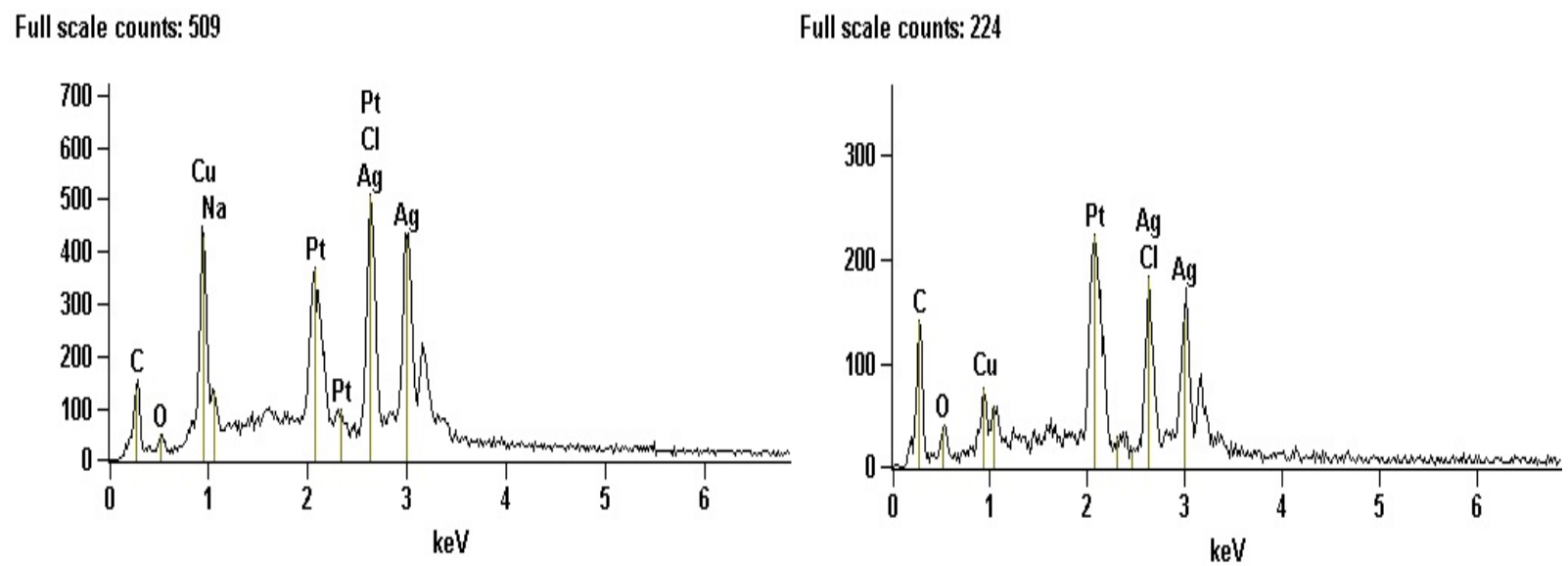

Fig. 5. EDS spectra of the biosynthesized AgCl NPs at $1 \mathrm{mM}$, and $3 \mathrm{Mm} \mathrm{AgNO}_{3}$, respectively.

In table 1, one can observe that silver chloride nanoparticles can be synthesized by different approaches, most of them starting from the $\mathrm{AgNO}_{3}$ like precursor. The difference consists in the type of stabilizer and surfactant.

The spherical shape is predominant in the case of silver chloride nanoparticles. Very closely to silver nanoparticles, as shown by the authors in [13], AgCl NPs present significant anti-microbial activity against Escherichia coli, Candida albicans, Staphylococcus Aureus.

The antimicrobial effect of colloidal silver chloride nanoparticles has been tested against different microorganisms, including fungi and bacteria. The results will be used for further investigation to determine the synergism of the samples in the presence of different antibiotics, how is shown in the paper "Characterization and antimicrobial activity of silver nanoparticles, biosynthesized using Bacillus species" [13].It is important to emphasize that the precursor used was $\mathrm{AgNO}_{3}$, a reason for which we have to take into account that the recipe for growth media used for the microorganisms is very important for the final results. For example, the Brain Heart Infusion used for growing the present fungi, because it contains sodium chloride, may be the main factor which has led to the silver chloride nanoparticles formation by reducing the precursor $\mathrm{AgNO}_{3}$ in the presence of biomass of Rhodotorula Mucilaginosa. 
Table 1. Characteristics and applications of AgCl NPs synthesized through different methods.

\begin{tabular}{|l|c|l|c|l|c|}
\hline Synthesis method & $\begin{array}{c}\text { Size } \\
{[\mathbf{n m}]}\end{array}$ & Shape & $\begin{array}{c}\text { Wavelength } \\
\text { max [nm] }\end{array}$ & Application & References \\
\hline $\begin{array}{l}\text { Green Synthesis } \\
\text { Streptomyces exfoliatus }\end{array}$ & $10-40$ & $\begin{array}{l}\text { Spherical } \\
\text { rod }\end{array}$ & 410 & $\begin{array}{l}\text { Antibacterial } \\
\text { Cytotoxicity: } \\
\text { HeLa and SiHa } \\
\text { cell lines }\end{array}$ & {$[2]$} \\
\hline $\begin{array}{l}\text { Green Synthesis } \\
\text { Bacillus subtilis }\end{array}$ & $20-60$ & spherical & 400 & Antifungal & [4] \\
\hline $\begin{array}{l}\text { Green Synthesis } \\
\text { Cissus quadrangularis } \\
\text { Linn }\end{array}$ & $15-23$ & spherical & 440 & Antibacterial & [5] \\
\hline $\begin{array}{l}\text { Green Synthesis } \\
\text { marine alga S. } \\
\text { plagiophyllum }\end{array}$ & $18-42$ & spherical & 417 & Antibacterial & [6] \\
\hline $\begin{array}{l}\text { Chemical synthesis } \\
\text { Precipitation }\end{array}$ & $6-7$ & hexagonal & - & Catalysis & [9] \\
\hline $\begin{array}{l}\text { Green Synthesis } \\
\text { Prunus persica L. outer } \\
\text { peel extract }\end{array}$ & $15-50$ & spherical & 440 & $\begin{array}{l}\text { Antibacterial, } \\
\text { Anticandidal, } \\
\text { Antioxidant }\end{array}$ & {$[8]$} \\
\hline $\begin{array}{l}\text { Chemical synthesis } \\
\text { One pot method }\end{array}$ & $\begin{array}{l}\text { Aroun } \\
\mathrm{d} 100\end{array}$ & $\begin{array}{l}\text { Spherical } \\
\text { ellipsoidal }\end{array}$ & 268 & Antibacterial & [12] \\
\hline
\end{tabular}

\section{Summary}

In the present work, silver chloride nanoparticles were synthesized via bio-reduction. It has been proved that the aqueous enzymatic extract of Rhodotorula Mucilaginosa fungi is able to synthesize silver chloride nanoparticles. It has been found that the silver chloride nanoparticles bioreduced by Rhodotorula Mucilaginosa decrease in size with increasing precursor concentration. The low-cost synthesis method is strengthened by the environmentally friendly steps of the procedure.

\section{References}

[1] X. Li, H. Xu, Z-S. Chen, G., Chen, Biosynthesis of nanoparticles by microorganisms and their applications, J. Nanomater. 2011 (2011), Article ID 270974, 16 pages.

[2] A.M. Iniyan et al., In vivo safety evaluation of antibacterial silver chloride nanoparticles from Streptomyces exfoliatus ICN25 in zebrafish embryos, Microb.Pathog., 112 (2017) 76 - 82. https://doi.org/10.1016/j.micpath.2017.07.054

[3] K. Paulkumar, et al., Eco-friendly synthesis of silver chloride nanoparticles using Klebsiella planticola (MTCC 2277), IJGCB, 3:1 (2013) 12 - 16.

[4] K. Paulkumar, et al., Biosynthesis of silver chloride nanoparticles using bacillus subtilis MTCC 3053 and assessment of its antifungal activity, 2013 (2013), Article ID 317963, 8 pages.

[5] V. Gopinath, et al., Biogenic synthesis of antibacterial silver chloride nanoparticles using leaf extracts of Cissus quadrangularis Linn, Mater. Lett. 91 (2013) 224 - 227. https://doi.org/10.1016/j.matlet.2012.09.102 
[6] T. Stalin Dhas, et al., Facile synthesis of silver chloride nanoparticles using marine alga and its antibacterial efficacy, Spectrochim. Acta Mol. Biomol.Spectrosc. 120 (2014) 416 - 420. https://doi.org/10.1016/j.saa.2013.10.044

[7] V.A. Kumar, et al., Synthesis of nanoparticles composed of silver and silver chloride for a plasmonic photocatalyst using an extract from needles of Pinus densiflora, Mater. Lett. 176 (2016) 169 - 172. https://doi.org/10.1016/j.matlet.2016.04.077

[8] J.K. Patra, K.H. Baek, Green synthesis of silver chloride nanoparticles using Prunus persica L. outer peel extract and investigation of antibacterial, anticandidal, antioxidant potential, Green Chemistry Letters and Reviews, 9:2 (2016) 132 - 142. https://doi.org/10.1080/17518253.2016.1192692

[9] W. Hu, et al., In situ synthesis of silver chloride nanoparticles into bacterial cellulose membranes, Mater. Sci. $\quad$ Eng. $\quad$ C. $29 \quad$ (2009) $1216 \quad$ - 1219. https://doi.org/10.1016/j.msec.2008.09.017

[10] M., Sophocleous, J.K. Atkinson, A review of screen-printed silver/silver chloride $(\mathrm{Ag} / \mathrm{AgCl})$ reference electrodes potentially suitable for environmental potentiometric sensors, Sen. Actuators A Phys. https://doi.org/10.1016/j.sna.2017.10.01.

[11] S. Majumder, Synthesis and characterization of surfactant stabilized nanocolloidal dispersion of silver chloride in aqueous medium, Colloids Surf. A. 443 (2014) 156 - 163. https://doi.org/10.1016/j.colsurfa.2013.10.064

[12] I. Ghiuta, et al., Characterization and antimicrobial activity of silver nanoparticles, biosynthesized using Bacillus species, Appl. Surf. Sci. 438 (2018) 66 - 73. https://doi.org/10.1016/j.apsusc.2017.09.163

[13] H. Ha, J. Payer, The effect of silver chloride formation on the kinetics of silver dissolution in chloride solution, Electrochim. Acta 56 (2011) 2781 - 2791. https://doi.org/10.1016/j.electacta.2010.12.050 